\title{
Combinatory action of VEGFR2 and MAP kinase pathways maintains endothelial-cell integrity
}

\author{
Hanbing Zhong ${ }^{1, *}$, Danyang Wang ${ }^{1, *}$, Nan Wang ${ }^{1}$, Yesenia Rios ${ }^{2}$, Haigen Huang ${ }^{2}$, Song $\mathrm{Li}^{1}$, Xinrong Wu ${ }^{3}$, \\ Shuo Lin ${ }^{1,2}$ \\ ${ }^{I}$ Laboratory of Chemical Genomics, School of Chemical Biology and Biotechnology, Peking University, Shenzhen Graduate \\ School, Shenzhen University Town, Shenzhen 518055, China; ${ }^{2}$ Department of Molecular, Cell and Developmental Biology, Univer- \\ sity of California, Los Angeles, Los Angeles, CA 90095, USA; ${ }^{3}$ Liu Hua Qiao Hospital, Guangzhou 510010, China
}

Blood vessels normally maintain stereotyped lumen diameters and their stable structures are crucial for vascular function. However, very little is known about the molecular mechanisms controlling the maintenance of vessel diameters and the integrity of endothelial cells. We investigated this issue in zebrafish embryos by a chemical genetics approach. Small molecule libraries were screened using live $T g(k d r l: G R C F P)^{z n l}$ transgenic embryos in which endothelial cells are specifically labeled with GFP. By analyzing the effects of compounds on the morphology and function of embryonic blood vessels after lumen formation, PP1, a putative Src kinase inhibitor, was identified as capable of specifically reducing vascular lumen size by interrupting endothelial-cell integrity. The inhibitory effect is not due to Src or general VEGF signaling inhibition because another Src inhibitor and Src morpholino as well as several VEGFR inhibitors failed to produce a similar phenotype. After profiling a panel of 22 representative mammalian kinases and surveying published data, we selected a few possible new candidates. Combinational analysis of these candidate kinase inhibitors established that PP1 induced endothelial collapse by inhibiting both the VEGFR2 and MAP kinase pathways. More importantly, combinatory use of two clinically approved drugs Dasatinib and Sunitinib produced the same phenotype. This is the first study to elucidate the pathways controlling maintenance of endothelial integrity using a chemical genetics approach, indicating that endothelial integrity is controlled by the combined action of the VEGFR2 and MAP kinase pathways. Our results also suggest the possible side effect of the combination of two anticancer drugs on the circulatory system.

Keywords: PP1; endothelial-cell integrity; vascular lumen; zebrafish

Cell Research (2011) 21:1080-1087. doi:10.1038/cr.2011.41; published online 22 March 2011

\section{Introduction}

The formation and maintenance of vascular lumens by endothelial cells are very important for the establishment of a functional vertebrate circulatory system. Vascular lumens are mainly formed by two means, endothelialcell hollowing or endothelial-cell-cord hollowing [1-4]. In vitro studies indicate that this process is regulated by

\footnotetext{
*These two authors contributed equally to this work. Correspondence: Shuo Lin

Tel: +1-310-267-4970; Fax: +1-310-267-4971

E-mail: shuolin@ucla.edu

Abbreviations: hours post fertilization (hpf); days post fertilization (dpf) Received 9 September 2010; revised 16 November 2010; accepted 14 December 2010; published online 22 March 2011
}

integrin- and cdc42/Rac1-dependent pinocytic events downstream of the integrin-extracellular matrix signaling pathway [5-7]. However, the mechanism of vascular lumen maintenance is still largely unknown [8].

The major challenge facing investigations into this question is that classic loss-of-function and gain-of-function genetic approaches are not applicable because the initial defects in vasculogenesis or angiogenesis usually impair lumen formation as well. Although conditional gene knockouts in mice may overcome this shortcoming, analysis of vessel diameter in live embryos is made difficult by in utero development. In this study, we used a transgenic zebrafish model coupled with a temporally controlled chemical genetics approach to elucidate the mechanism of vascular lumen maintenance. Vascular development of zebrafish embryos has been well studied 
$[9,10]$. The large axial vessels (dorsal aorta and posterior cardinal vein) and caudal plexus are formed by vasculogenesis while other vessels are formed by angiogenesis. By $24 \mathrm{hpf}$ (hours post fertilization), the heart begins to beat and blood cells circulate in the axial vessels. Furthermore, unlike mammal models, zebrafish embryos can survive and develop for up to about 1 week without any blood circulation owing to its small body that is readily accessible to the diffusion of oxygen, $\mathrm{CO}_{2}$, and nutrients [11]. This ability offers a unique opportunity to examine lumen maintenance defects that usually cause lethality in mammals. In addition, several endothelial-cell-specific GFP transgenic zebrafish lines have been generated with VEGFR2 (also known as flk or kdrl) or flila promoters, which render easy, fast, and continuous observation of blood vessel development under a fluorescence microscope [12].

Small-molecule chemicals offer easier and more precise temporal control of gene function by allowing the addition and removal of a given compound at preselected time points. A chemical capable of specifically modifying a biological process is not only a useful molecular and biological tool but also a potential drug candidate. Zebrafish has been used for chemical library screens, including antiangiogenesis screens, but most of them have been conducted by adding compounds as early as $2 \mathrm{hpf}$ or at prevasculogenesis or angiogenesis stages. To identify small molecules that can specifically regulate the maintenance of the vascular system, we performed a screen using two libraries consisting of $\sim 1700$ chemical compounds by adding each compound to embryos placed in 96-well plates at $30 \mathrm{hpf}$, when blood circulation is well established, and examined changes in blood circulation at $48 \mathrm{hpf}$. One of the compounds, PP1, a previously known inhibitor of Src kinase, stopped circulation but did not have any morphological effect on the whole embryo. Further examination of $T g(k d r l: G R C F P)^{z n l}$ transgenic embryos treated by PP1 revealed that blood vessels, in particular the dorsal aorta and intersegmental vessels (ISV), had reduced or closed vessel lumens through endothelialcell collapse. We show here that pathways triggered by PP1 in the context of blood vessel lumen regulation are not Src-dependent but rather involve a combinatory action of VEGFR and MAP kinase signaling pathways.

\section{Results}

Identification and characterization of PP1 as a molecule that regulates vascular lumen maintenance

Through screening $\sim 1700$ small-molecule compounds of the BioMol and Prestwick libraries, PP1 was identified as one of the compounds that stopped blood circu- lation but had no effect on heart beat and overall body morphology (Figure 1 and supplementary information, Figure S1). Angiography with a $2000-\mathrm{kDa}$ green fluorescent dye showed that the dye was completely blocked in the heart and did not enter the blood vessels (Figure 1D). To further reveal the activity of PP1 in regulating blood vessel formation as well as function, it was added to embryos at the shield, $30 \mathrm{hpf}, 2 \mathrm{dpf}, 3 \mathrm{dpf}$, and $4 \mathrm{dpf}$ stages. When added at the shield stage, PP1 inhibited the growth of intersegmental vessel in a dose-dependent manner, completely blocking ISV sprouting at $5 \mu \mathrm{M}$ concentration (Supplementary information, Figure S2). When added at later stages of $30 \mathrm{hpf}, 2 \mathrm{dpf}, 3 \mathrm{dpf}$, and $4 \mathrm{dpf}, 10$ $\mu \mathrm{M}$ PP1 could completely block the already-established circulation after about $16 \mathrm{~h}$ of treatment (Supplementary information, Movies S1 and S2).

To study the unique activity of PP1 in regulating the late maintenance of blood vessels, we chose to add it at $3 \mathrm{dpf}$ and observe the phenotype at $4 \mathrm{dpf}$. Lateral-view images taken by confocal microscopy showed that the dorsal aorta of PP1-treated embryos was thinner than that of the control, implying lumen size reduction (Figure 2B). Cross sections confirmed that PP1 indeed caused vascular lumens to reduce. In PP1-treated embryos, both the dorsal aorta and cardinal vein were narrower, with

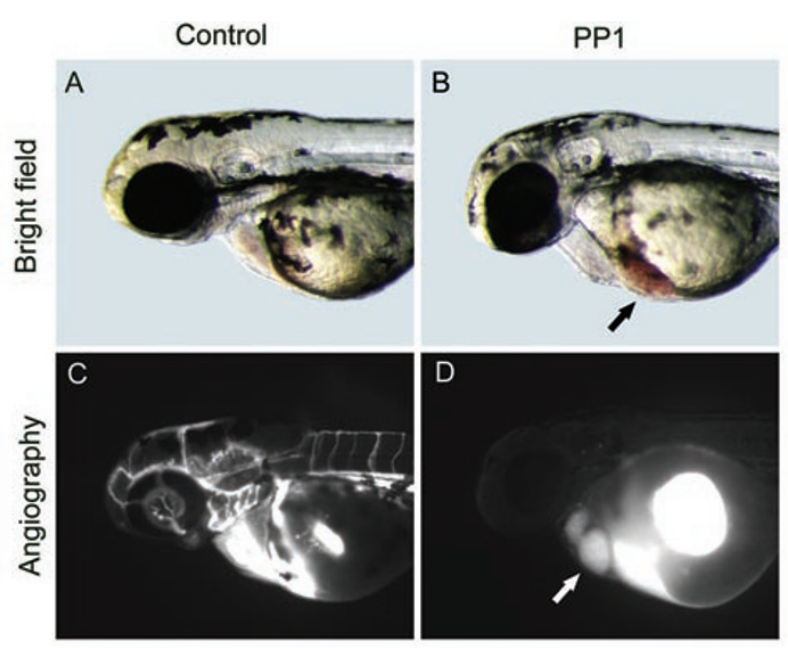

Figure 1 PP1 blocked established blood circulation. PP1 was added to embryos at $30 \mathrm{hpf}$ when blood circulation was already established. At $48 \mathrm{hpf}$ the blood circulation of PP1-treated embryos stopped. (A and C) Control embryos. (B and D) PP1treated embryos. Except blood cells stuck at sinus venous (black arrow), PP1-treated embryos looked normal. (C) Fluorescein isothiocyanate dextran ( $\mathrm{MW}=2000000 \mathrm{Da})$ was observed in circulation, labeling the whole vasculature. (D) Fluorescein isothiocyanate dextran was stuck in heart (white arrow), indicating the lumen of dorsal aorta reduced and did not allow the dye to pass. 


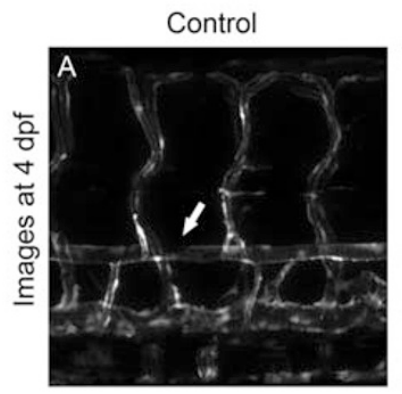

PP1 $10 \mu \mathrm{M}$ at $3 \mathrm{dpf}$

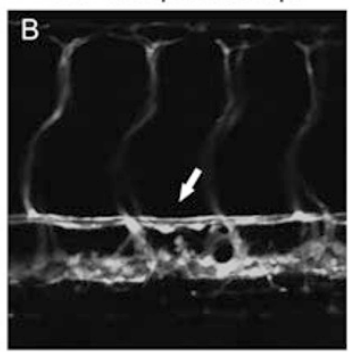

SU6656 $20 \mu \mathrm{M}$ at $3 \mathrm{dpf}$

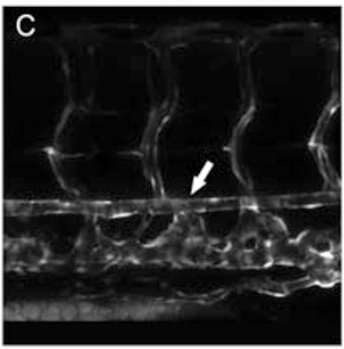

Sunitinib $20 \mu \mathrm{M}$ at $3 \mathrm{dpf}$

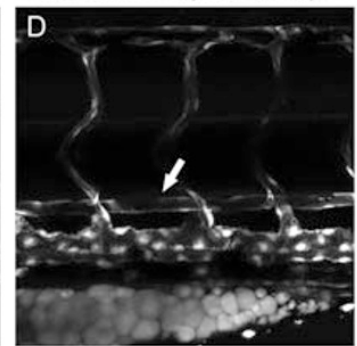

Figure 2 PP1 caused dorsal aorta to reduce while SU6656 and Sunitinib did not. All these three small molecules were added to $T g(k d r l: G R C F P)^{z n 1}$ embryos at $3 \mathrm{dpf}$ and images were taken at $4 \mathrm{dpf}$. The trunk region above yolk extension was shown. White arrows point to the dorsal aorta. In PP1-treated embryos the dorsal aorta looked thinner than in control. In SU6656- or Sunitinib-treated embryos, the dorsal aortas remained the same.

the dorsal aorta shrinking more severely. The lumen of the dorsal aorta was almost absent (Figure 3B and 3F). The blockage of blood vessels was further demonstrated by microangiography with tetramethylrhodamine dextran $(2000 \mathrm{kDa})$ in $T g(k d r l: G R C F P)^{z n l}$ embryos. In PP1treated embryos, tetramethylrhodamine dextran failed to enter circulation (Supplementary information, Figure S3). To rule out the possibility that the absence of blood flow and blood pressure could lead to collapse of vascular lumens, a myosin-ATPase inhibitor BDM (2, 3-butanedione 2-monoxime) was used to stop the heart beat of 3 dpf zebrafish embryos [13]. After incubation of embryos in BDM for $24 \mathrm{~h}$, vascular lumen appeared intact in the absence of heart beat (Supplementary information, Figure S4). Moreover, PP1 treatment did not stop heart beat. Together, these data indicate that lack of blood circulation caused by PP1 was the consequence of the reduction of vascular lumens.

PP1 did not appear to kill endothelial cells as the green fluorescence of either $\operatorname{Tg}(k d r l: G R C F P)^{z n l}$ or $\operatorname{Tg}(\text { flila: } n E G F P)^{y 7}$ embryos remained intact, suggesting the presence of live endothelial cells. To further confirm this conclusion, whole mount TUNEL assay was performed with DMSO (vehicle control) or PP1-treated
Control

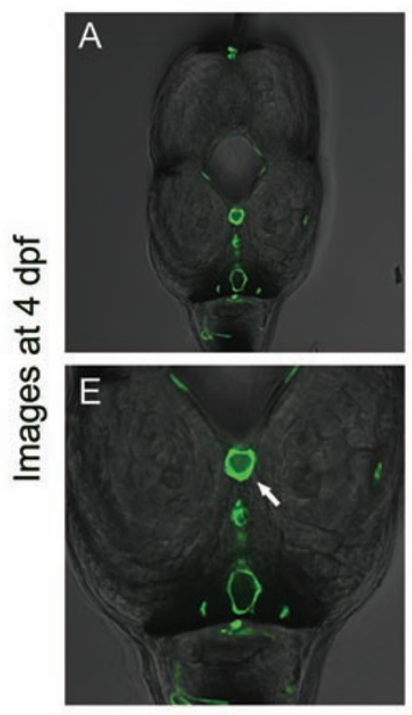

PP1 $10 \mu \mathrm{M}$ at $3 \mathrm{dpf}$
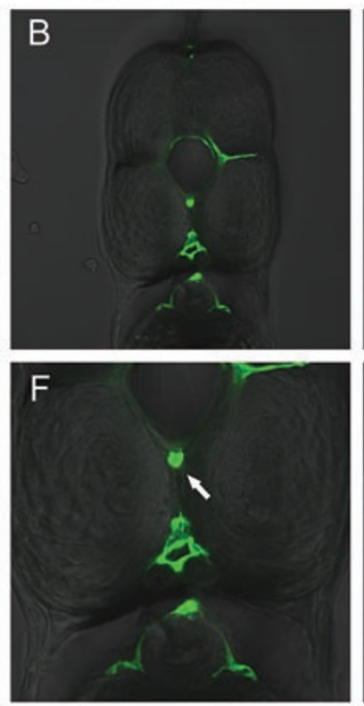

\section{SU6656 $20 \mu \mathrm{M}$ at $3 \mathrm{dpf}$ Sunitinib $20 \mu \mathrm{M}$ at $3 \mathrm{dpf}$}
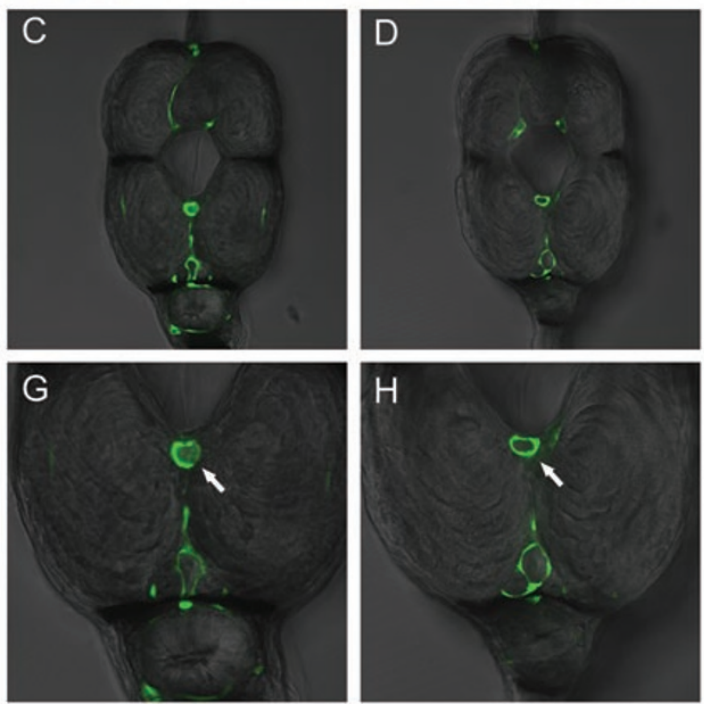

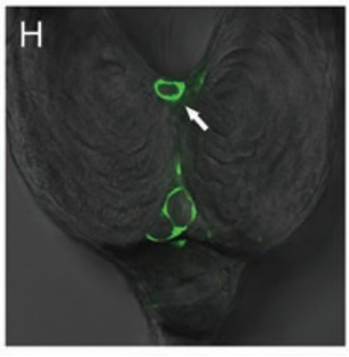

Figure 3 Cross section showed PP1 did cause vascular lumens to collapse. The lower row is the magnification of upper row. White arrows point to the lumen of dorsal aorta. In PP1-treated embryos, both the dorsal aorta and cardinal vein collapsed, while the dorsal aorta collapsed more severely than the cardinal vein. The lumen of dorsal aorta disappeared. In other groups, the dorsal aorta and cardinal vein remained intact. 
$\operatorname{Tg}(\text { flila:nEGFP })^{y 7}$ embryos and no difference in apoptosis of endothelial cells was observed (Supplementary information, Figure S5). Under the same PP1 treatment condition, the pronephric duct lumen appeared intact (Supplementary information, Figure S6), indicating that the activity of PP1 on endothelial cells was specific.

To examine structural changes in endothelial-cell morphology induced by PP1 administration, we analyzed blood vessels by electron microscopy. Compared to the control embryos at $4 \mathrm{dpf}$ (Figure 4A), the PP1-treated embryos exhibited non-functional aortic lumens and no blood cells in the dorsal aorta (Figure 4B). The diameter of the aortic lumen was smaller than a red blood cell (Figure 4B), consequently prohibiting circulation. The endothelial cells in dorsal aorta, posterior cardinal vein, and ISVs in PP1-treated embryos were collapsed (Figure 4 and Supplementary information, Figures S6 and S7). In the control embryos, they were squamous shaped (Figure 4C), whereas in PP1-treated embryos, they were shorter and thicker, and lacked tight adhesions with surrounding cells (Figure 4D). The cell junctions between

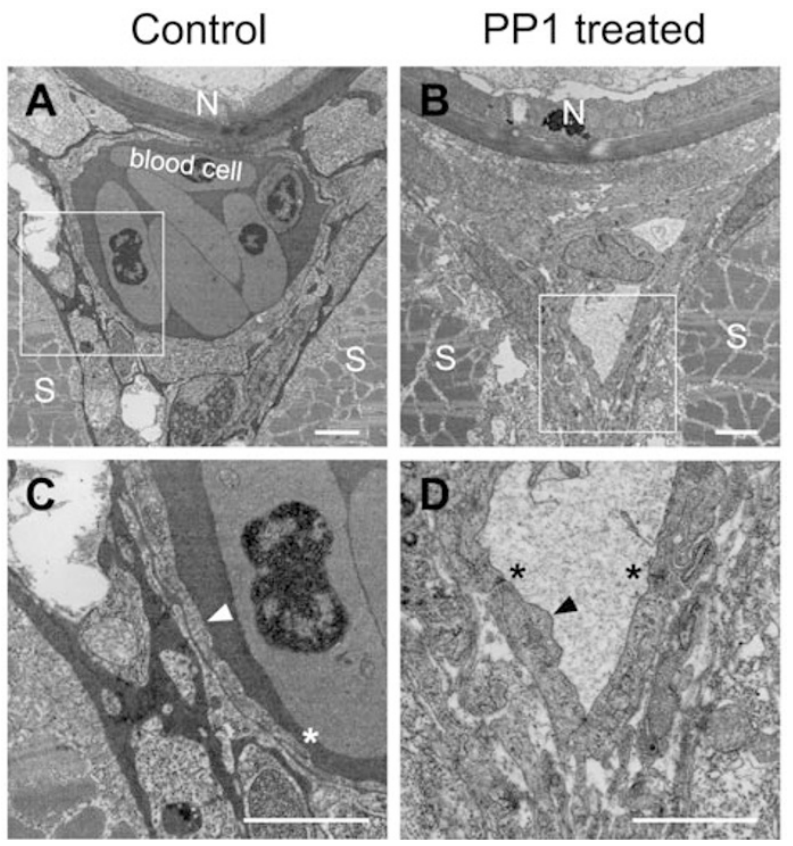

Figure 4 The ultra-structural changes of endothelial cells in aorta revealed by electron microscopy. The images were transverse sections with dorsal to the top. The lumen changes between the control embryos (A) and the PP1-treated embryos (B) at 4 dpf. (C and D) The structural changes of endothelial cells boxed in A and B, respectively. Note that the endothelial cell in D (black arrow head) was short and thick compared with that in $\mathbf{C}$ (white arrow head). The stars indicated the cell junctions between endothelial cells. N, notochord; S, somite. Scale bars are $2 \mu \mathrm{m}$. the endothelial cells were also abnormal in PP1-treated embryos (Figure 4D, black stars) compared with control embryos (Figure 4C, white star). The inhibitory activities of PP1 were not reversible as circulation failed to recover in PP1-treated embryos after washing with fresh fish water.

To investigate if PP1 could also cause vascular lumen regression in mammalian systems, we added it to established network of HUVEC (human umbilical vascular endothelial cell) in Matrigel. As shown in Supplementary information, Figure S8, the HUVEC network was disrupted in a dose-dependent manner.

Vessel lumen reduction by PP1 is neither through Src kinase nor VEGF pathway alone

PP1 was initially reported as an ATP-competitive Src kinase inhibitor [14]. To study the mode of action of PP1 in zebrafish, we first compared it with SU6656 (Supplementary information, Figure S1), the most selective Src kinase inhibitor reported so far $[15,16]$. Unexpectedly, we did not observe any lumen diameter reduction induced by SU6656, suggesting a non-Src mechanism (Figures $2 \mathrm{C}, 3 \mathrm{C}$ and $3 \mathrm{G}$ ). To confirm this idea, we knocked down the zebrafish Src gene by injecting morpholino that specifically blocks the translation of the Src protein [17]. Again, no lumen diameter reduction was observed. Since PP1 inhibited angiogenic ISV formation when added earlier at the shield stage, which can also be caused by inhibiting VEGF signaling (Supplementary information, Figure S2) [12], we tested if Sunitinib [18, 19], a kinase inhibitor that has strong activity on multiple VEGFRs could also reduce lumen size like PP1 when added at late stages. As shown in Figures 2 and 3, Sunitinib did not produce the same defective phenotype as PP1 did. These studies suggest that PP1 has activity in regulating other targets required for maintaining blood vessel lumens.

\section{Combined action of VEGFR and MAP kinase pathways maintains vessel lumen integrity}

The ATP-analogous structure of PP1 suggests that its primary targets are most likely kinases. To explore other kinases inhibited by PP1 as potential targets involved in blood vessel lumen maintenance, we profiled its activity against a panel of 22 representative kinases. As shown in Table 1, PP1 inhibited ABL1 $(89 \%$ at $5 \mu \mathrm{M})$, LCK $(93 \%$ at $5 \mu \mathrm{M})$, and VEGFR2 $(92 \%$ at $5 \mu \mathrm{M})$. As mentioned above, inhibition of VEGFR 2 alone failed to reproduce the phenotype of late PP1 treatment. It has been previously reported that PP1 has multiple targets [20], as confirmed here in our kinase profiling study. We therefore tested the combinatory action of these potential candidates using different selective kinase inhibitors. 
Table 1 PP1 kinase profiling

\begin{tabular}{lclc}
\hline Kinase & Inhibition (\%) & \multicolumn{1}{c}{ Kinase } & Inhibition (\%) \\
\hline ABL1 & 89 & MAP2K1 (MEK1) & 20 \\
CAMK1D (CaMKI delta) & 6 & MAP3K9 (MLK1) & 29 \\
CAMK2B (CaMKII beta) & -1 & MAPK1 (ERK2) & 3 \\
CDK2/cyclin A & 5 & MAPK8 (JNK1) & 7 \\
CHEK2 (CHK2) & 8 & MAPKAPK2 & 2 \\
CLK1 & 10 & MYLK2 (skMLCK) & 5 \\
CSNK1D (CK1 delta) & 68 & NEK2 & 2 \\
FLT3 & 56 & PLK1 & -1 \\
GSK3B (GSK3 beta) & 0 & RPS6KA1 (RSK1) & 9 \\
KDR (VEGFR2) & 92 & SGK (SGK1) & 4 \\
LCK & 93 & STK3 (MST2) & 13 \\
\hline
\end{tabular}

However, combined testing of ABL1, LCK and VEGFR2 using specific chemical inhibitors (Bcr-abl Inhibitor for ABL1, SU6656 for LCK, and Sunitinib for VEGFR2) did not reproduce the same phenotype of PP1 treatment ( $n=60$, embryos treated at $3 \mathrm{dpf}$, none showed block of circulation).

It has been noticed that PP1 can inhibit the MAP kinase pathway in HUVEC [21]. In another study, anthrax toxin, whose targets include MEK1/2 [22], appeared to reduce vessel lumen size in zebrafish [23]. During earlier developmental stages, MEK1/2 is also involved in artery specification in both zebrafish and mice [24, 25]. Together these findings imply that MEK $1 / 2$ may cooperate with VEGFR to regulate blood vessel lumen diameter. We examined this hypothesis by combining VEGFR inhibitor Sunitinib and MEK1/2 inhibitor U0126. Indeed, U0126 $(10 \mu \mathrm{M})$ combined with Sunitinib $(20 \mu \mathrm{M})$ resulted in a reduction of vessel lumen size (Figure 5F), whereas individually they did not. Combined treatment of another MAP kinase signaling pathway inhibitor Dasatinib (20 $\mu \mathrm{M})$ with Sunitinib $(20 \mu \mathrm{M})$ produced a similar phenotype as PP1, whereas each individual inhibitor did not result in any vessel shrinkage even at much higher concentrations (Figure 5C).

Since there are three major VEGF receptors, it is desirable to determine which receptor is involved in combinatory action with MEK1/2. To address this issue, additional highly selective VEGFR inhibitors PTK787 and ZM323881 were tested. Both of these inhibitors (PTK787 at $5 \mu \mathrm{M}$ or ZM323881 at $5 \mu \mathrm{M}$ ), when individually combined with Dasatinib or U0126, induced a reduction of vessel lumen size (Figure 5D, 5E, 5G, and $5 \mathrm{H})$. However, they did not lead to this phenotype when added alone. Given that PTK787 does not efficiently inhibit VEGFR3 [26] and ZM323881 is inactive against VEGFR1 [27, 28], VEGFR2 is therefore the most likely factor involved in maintaining lumen size. Collectively, our results indicate that PP1 may induce reduction of the blood vessel lumen size by inhibiting both the VEGFR2 and MAP kinase pathways.

\section{Discussion}

To the best of our knowledge, this study represents the first analysis of mechanistic pathways involved in maintaining blood vessel lumen diameter after the establishment of functional circulation. The temporal chemical genetics approach coupled with transgenic zebrafish technology offers a novel entry point toward analyzing this late function of blood vessels. Through testing PP1 at a series of developmental stages, we establish that adding compounds to embryos at $3 \mathrm{dpf}$ and examining their effect at $4 \mathrm{dpf}$ is an effective protocol to test candidates involved in regulating the maintenance of endothelial cells. With circulation fully established and the dorsal aorta and posterior cardinal vein separated completely after 3 dpf, observation of circulation is very achievable. When added to embryos at $3 \mathrm{dpf}$, PP1 specifically caused endothelial cells to lose their integrity. It will be interesting to further analyze endothelial cell junction with antibody staining but nonetheless the present electron microscopic data suggest that the junctions between endothelial cells were dramatically changed by PP1 administration (Figure 4D).

As reported previously and confirmed by kinase profiling performed in this study, PP1 is an ATP-competitive kinase inhibitor targeting multiple kinases including $\mathrm{Src}$ family members, Abl, and VEGFR. Numerous studies have shown that the Src family and VEGFR are involved in vascular development, especially the VEGF pathway, which is the master regulator of angiogenesis. However, analysis with highly specific small-molecule inhibitors 

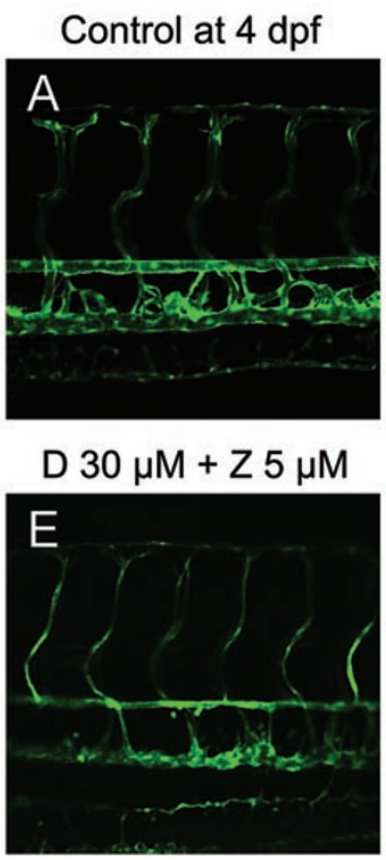
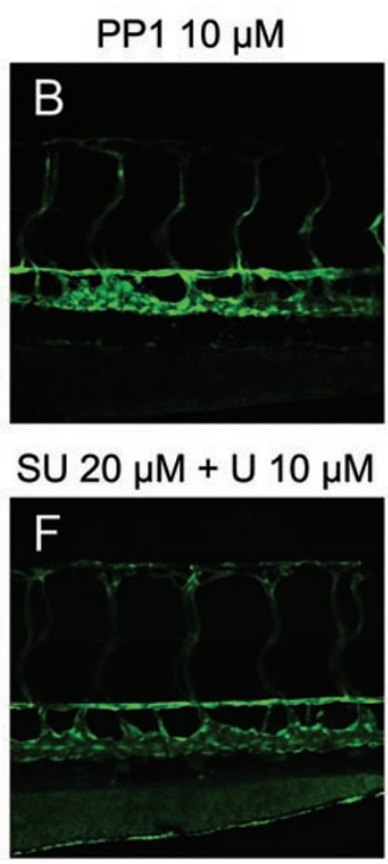
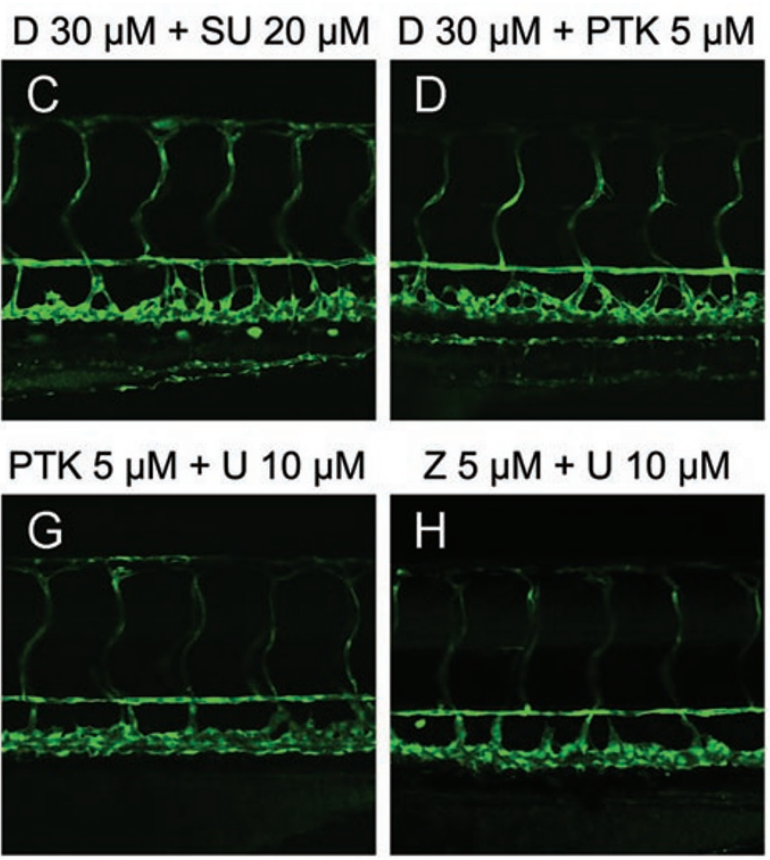
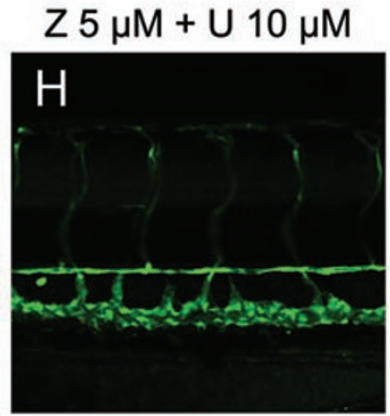

Figure 5 Combinational treatment of kinase inhibitors induces the similar phenotype produced by PP1. All images are lateral view with dorsal to the top and anterior to the left. The combinational treatment of Dasatinib (D) or U0126 (U) with Sunitinib (SU), PTK787 (PTK), or ZM323881 (Z) resulted in the shrinkage of dorsal aorta.

targeting the Src family or VEGFR showed that the application of each alone does not cause endothelial cells to collapse, implying additional unknown targets in the mode of PP1 action. We show here that activity of MEK1/2 (MAP2K) in collaboration with VEGFR2 is involved in the maintenance of endothelial cells. Since PP1 does not directly inhibit MAP2K in vitro (Table 1), it may exert its effect on endothelial cells through regulating other factors that consequently result in the reduction of MAP2K in vivo.

Since the mechanisms of endothelial-cell maintenance are likely conserved in humans, our findings on Dasatinib and Sunitinib may have clinical implications. Dasatinib (marketed as Sprycel by Bristol-Myers Squibb) is a dual ABL and Src family kinase inhibitor approved by the FDA for patients with imatinib-resistant chronic myelogenous leukemia and Philadelphia chromosome-positive acute lymphoblastic leukemia. Sunitinib (marketed as Sutent by Pfizer) is a multi-target receptor tyrosine kinase inhibitor approved by the FDA for the treatment of renal-cell carcinoma and imatinib-resistant gastrointestinal stromal tumor. Since the combined treatment of Dasatinib and Sunitinib caused vascular lumen reduction and circulation blockage, potential side effects should be considered if these two drugs are used together.

\section{Materials and Methods}

\section{Zebrafish stocks and chemical library screening}

The wild-type AB strain zebrafish and transgenic lines $T g(k d r l: G R C F P)^{z n 1}, \operatorname{Tg}(\text { flila:nEGFP })^{y^{7}}$, and $\operatorname{Tg}(\text { gata1:dsRed })^{s d 2}$ were used in this study. Embryos were raised under standard condition and staged according to description by Kimmel et al. [29]. Live embryos were placed into 96-well plates, 6 embryos per well with $200 \mu 1$ fresh fish water containing $1 \times$ Antibiotic-Antimycotic Solution (Mediatech, Manassas, VA). Chemical libraries were added to the embryos at the concentration of approximately $10 \mu \mathrm{M}$ at $30 \mathrm{hpf}$, and circulation and morphology of the embryos were observed at $48 \mathrm{hpf}$.

\section{Chemical libraries and compounds}

BIOMOL chemical library consisting of $\sim 500$ compounds of bioactive lipids, endocannabinoid, ion-channel ligands, enzyme inhibitors, phosphatase and kinase inhibitors, and orphan ligands (BIOMOL); and Prestwick library of 1120 compounds consisting of $85 \%$ FDA-approved drugs (Prestwick Chemical, Inc) were screened for compounds that regulate blood vessel function after circulation was established. PP1 was purchased from BIOMOL; Dasatinib, PTK787 and Sunitinib were purchased from Selleck; U0126 and BDM were purchased from Sigma-Aldrich; ZM323881 was purchased from Tocris; SU6656 and Bcr-abl Inhibitor were purchased from Calbiochem. High-concentration stocks of these organic compounds were made in DMSO. Working solutions were diluted from DMSO stocks into fish water. 
TUNEL assay

Apoptosis in the endothelial cells of $\operatorname{Tg}(\text { flila:nEGFP })^{y 7}$ transgenic embryos was examined using terminal transferase-mediated dUTP nick end-labeling (TUNEL) assay as per manufacturer's protocol with some modifications (In situ Cell Death Detection Kit, TMR Red, Roche Applied Science). After PP1 treatment, 4 dpf embryos were fixed overnight in $2 \%$ paraformaldehyde in PBS at $4{ }^{\circ} \mathrm{C}$. Embryos were then washed with PBST buffer twice and stored in methanol at $-20^{\circ} \mathrm{C}$ overnight. Embryos were rehydrated, permeabilized by proteinase K (Sigma) $(50 \mu \mathrm{g} / \mathrm{ml})$ for $40 \mathrm{~min}$, and refixed in $2 \%$ paraformaldehyde in PBS for $10 \mathrm{~min}$ at room temperature. Embryos were then washed $3 \times 5 \mathrm{~min}$ in PBST and incubated in the TUNEL reaction mix for $3 \mathrm{~h}$ at $37^{\circ} \mathrm{C}$ in darkness. After reaction, embryos were washed $3 \times 30 \mathrm{~min}$ in $\mathrm{PBST}$ at room temperature and stored in PBST at $4{ }^{\circ} \mathrm{C}$.

Tubular network degeneration assay with HUVEC

Matrigel (growth factor reduced) was thawed at $4{ }^{\circ} \mathrm{C}$ overnight, and each well of prechilled 96-well plates was coated with $50 \mu 1$ PBS-diluted Matrigel $(1: 1)$ and incubated at $37{ }^{\circ} \mathrm{C}$ for $30 \mathrm{~min}$. HUVECs ( 15000 cells per well) were added into each Matrigeltreated well and cultured in $0.1 \mathrm{ml} \mathrm{ECM}$ (supplemented with $0.5 \%$ FBS and $40 \mathrm{ng} / \mathrm{ml} \mathrm{VEGF).} \mathrm{After} \mathrm{incubation} \mathrm{at} 37{ }^{\circ} \mathrm{C}$ and $5 \% \mathrm{CO}_{2}$ for $4 \mathrm{~h}$, tube-like structures were formed, and then treated by PP1 at different concentrations for $18 \mathrm{~h}$. The density of tubular structure was quantified by manual counting of the length of endothelial network in high-power fields $(200 \times)$.

\section{Kinase profiling}

A panel of 22 representative mammalian kinases was tested for inhibition by PP1 using SelectScreen Kinase Profiling Service (Invitrogen, Carlsband, CA). The concentration of PP1 tested was $5 \mu \mathrm{M}$ in $1 \%$ DMSO.

\section{Microangiography}

Fluorescein isothiocyanate dextran $(\mathrm{MW}=2000000 \mathrm{Da}$, Sigma) or tetramethylrhodamine dextran (MW=2 $000000 \mathrm{Da}$, Invitrogen) dissolved in double-distilled water was microinjected into the sinus venous of zebrafish embryos at $48 \mathrm{hpf}$ or $4 \mathrm{dpf}$, respectively.

\section{Light microscopy}

Pictures of zebrafish embryos were taken with either a confocal microscope (Zeiss LSM510 Meta and Axiovert 200M), or AxioImager A1 microscope and AxioCam digital camera (Zeiss, Oberkochen, Germany), and edited with Photoshop 7.0 (Adobe Systems, San Jose, CA).

\section{Electron microscopy}

Embryos for the transmission electron microscopy were fixed in $2 \%(\mathrm{v} / \mathrm{v})$ glutaraldehyde (Sigma-Aldrich) and $2 \%(\mathrm{w} / \mathrm{v})$ paraformaldehyde (Sigma-Aldrich) dissolved in $0.1 \mathrm{M}$ sodium cacodylate buffer ( $\mathrm{pH} 7.4)$ at $4{ }^{\circ} \mathrm{C}$ overnight, and post-fixed in $2 \%(\mathrm{w} /$ v) osmium tetroxide at room temperature for $4 \mathrm{~h}$. After dehydrating through serial ethanol $(15 \%, 30 \%, 50 \%, 75 \%, 85 \%, 95 \%$, and $100 \%$ ), the embryos were embedded in Spurr's resin (SPI-Chem). Sections of $75 \mathrm{~nm}$ were obtained with a microtome (Leica), and stained with $1 \%(\mathrm{w} / \mathrm{v})$ uranyl acetate and $0.5 \%(\mathrm{w} / \mathrm{v})$ lead citrate. Images were obtained with an electron microscope (JEOL).

\section{Acknowledgments}

We thank Zahra Tehrani for editing the manuscript. This work was supported by research grants from the Peking University Shenzhen Graduate School (to Hanbing Zhong), the Project 31071281 supported by National Natural Science Foundation of China (to Hanbing Zhong), the 973 Program from MOST of China (2009CB941203 to Hanbing Zhong) and (2009CB940904 to Song Li), and the National Institutes of Health (R01 DK54508 to Shuo Lin).

\section{References}

1 Kamei M, Saunders WB, Bayless KJ, Dye L, Davis GE, Weinstein BM. Endothelial tubes assemble from intracellular vacuoles in vivo. Nature 2006; 442:453-456.

2 Blum Y, Belting HG, Ellertsdottir E, Herwig L, Lüders F, Affolter M. Complex cell rearrangements during intersegmental vessel sprouting and vessel fusion in the zebrafish embryo. Dev Biol 2008; 316:312-322.

3 Ellertsdottir E, Lenard A, Blum Y, et al. Vascular morphogenesis in the zebrafish embryo. Dev Biol 2010; 341:56-65.

4 Strilic B, Kucera T, Eglinger J, et al. The molecular basis of vascular lumen formation in the developing mouse aorta. Dev Cell 2009; 17:505-515.

5 Bayless KJ, Salazar R, Davis GE. RGD-dependent vacuolation and lumen formation observed during endothelial cell morphogenesis in three-dimensional fibrin matrices involves the alpha(v)beta(3) and alpha(5)beta(1) integrins. Am J Pathol 2000; 156:1673-1683.

6 Bayless KJ, Davis GE. The Cdc42 and Rac1 GTPases are required for capillary lumen formation in three-dimensional extracellular matrices. J Cell Sci 2002; 115(Pt 6):1123-1136.

7 Davis GE, Bayless KJ. An integrin and Rho GTPase-dependent pinocytic vacuole mechanism controls capillary lumen formation in collagen and fibrin matrices. Microcirculation 2003; 10:27-44.

8 Iruela-Arispe ML, Davis GE. Cellular and molecular mechanisms of vascular lumen formation. Dev Cell 2009; 16:222231.

9 Isogai S, Horiguchi M, Weinstein BM. The vascular anatomy of the developing zebrafish: an atlas of embryonic and early larval development. Dev Biol 2001; 230:278-301.

10 Childs S, Chen JN, Garrity DM, Fishman MC. Patterning of angiogenesis in the zebrafish embryo. Development 2002; 129:973-982.

11 Stainier DY, Weinstein BM, Detrich HW 3rd, Zon LI, Fishman MC. Cloche, an early acting zebrafish gene, is required by both the endothelial and hematopoietic lineages. Development 1995; 121:3141-3150.

12 Cross LM, Cook MA, Lin S, Chen JN, Rubinstein AL. Rapid analysis of angiogenesis drugs in a live fluorescent zebrafish assay. Arterioscler Thromb Vasc Biol 2003; 23:911-912.

13 Nicoli S, Standley C, Walker P, Hurlstone A, Fogarty KE, Lawson ND. MicroRNA-mediated integration of haemodynamics and Vegf signalling during angiogenesis. Nature 2010; 464:1196-1200.

14 Hanke JH, Gardner JP, Dow RL, et al. Discovery of a novel, potent, and Src family-selective tyrosine kinase inhibitor. 
Study of Lck- and FynT-dependent T cell activation. J Biol Chem 1996; 271:695-701.

15 Blake RA, Broome MA, Liu X, et al. SU6656, a selective src family kinase inhibitor, used to probe growth factor signaling. Mol Cell Biol 2000; 20:9018-9027.

16 Courtneidge SA. Role of Src in signal transduction pathways. The Jubilee Lecture. Biochem Soc Trans 2002; 30:11-17.

17 Jopling C, den Hertog J. Fyn/Yes and non-canonical Wnt signalling converge on RhoA in vertebrate gastrulation cell movements. EMBO Rep 2005; 6:426-431.

18 Karaman MW, Herrgard S, Treiber DK, et al. A quantitative analysis of kinase inhibitor selectivity. Nat Biotechnol 2008; 26:127-132.

19 Roskoski R Jr. Sunitinib: a VEGF and PDGF receptor protein kinase and angiogenesis inhibitor. Biochem Biophys Res Commun 2007; 356:323-328.

20 Apsel B, Blair JA, Gonzalez B, et al. Targeted polypharmacology: discovery of dual inhibitors of tyrosine and phosphoinositide kinases. Nat Chem Biol 2008; 4:691-699.

21 Merhi-Soussi F, Dominguez Z, Macovschi O, et al. Mechanisms involved in the stimulation of prostacyclin synthesis by human lymphocytes in human umbilical vein endothelial cells. Br J Pharmacol 2003; 139:321-328.

22 Duesbery NS, Webb CP, Leppla SH, et al. Proteolytic inactivation of MAP-kinase-kinase by anthrax lethal factor. Science 1998; 280:734-737.

23 Bolcome RE 3rd, Sullivan SE, Zeller R, Barker AP, Collier
RJ, Chan J. Anthrax lethal toxin induces cell death-independent permeability in zebrafish vasculature. Proc Natl Acad Sci US A 2008; 105:2439-2444.

24 Hong CC, Peterson QP, Hong JY, Peterson RT. Artery/vein specification is governed by opposing phosphatidylinositol-3 kinase and MAP kinase/ERK signaling. Curr Biol 2006; 16:1366-1372.

25 Ren B, Deng Y, Mukhopadhyay A, et al. ERK1/2-Akt1 crosstalk regulates arteriogenesis in mice and zebrafish. $J$ Clin Invest 2010; 120:1217-1228.

26 Wood JM, Bold G, Buchdunger E, et al. PTK787/ZK 222584, a novel and potent inhibitor of vascular endothelial growth factor receptor tyrosine kinases, impairs vascular endothelial growth factor-induced responses and tumor growth after oral administration. Cancer Res 2000; 60:2178-2189.

27 Endo A, Fukuhara S, Masuda M, Ohmori T, Mochizuki N. Selective inhibition of vascular endothelial growth factor receptor-2 (VEGFR-2) identifies a central role for VEGFR-2 in human aortic endothelial cell responses to VEGF. $J$ Recept Signal Transduct Res 2003; 23:239-254.

28 Whittles CE, Pocock TM, Wedge SR, et al. ZM323881, a novel inhibitor of vascular endothelial growth factor-receptor-2 tyrosine kinase activity. Microcirculation 2002; 9:513522.

29 Kimmel CB, Ballard WW, Kimmel SR, Ullmann B, Schilling TF. Stages of embryonic development of the zebrafish. Dev Dyn 1995; 203:253-310.

(Supplementary information is linked to the online version of the paper on the Cell Research website) 